\title{
Bonanza of New Treatment Regimens for Multiple Myeloma: What Is Right for My Patient?
}

Presented by Shaji K. Kumar, MD

\section{ABSTRACT}

Although recent advances in the treatment of multiple myeloma have improved survival, it remains a chronic disease that requires a long-term treatment strategy. The key to achieving the best outcomes for patients is delivering the best "package" of treatment at a given stage. This means using optimal combinations that maximize benefit based on what patients have received previously and minimize treatment-related toxicity. Sequencing of regimens also plays an important role. As new agents and new classes of drugs continue to be approved for multiple myeloma, future strategies will use more individualized approaches to treatment.

Therapeutic advances have led to prolonged survival in multiple myeloma (MM), but it remains a chronic disease. The addition of several new therapies for both newly diagnosed and relapsed disease has also added complexity to treatment algorithms, noted Shaji K. Kumar, MD, Hematologist, Professor of Medicine, and Chair of the Myeloma Group at Mayo Clinic Cancer Center, during the NCCN 2020 Virtual Congress: Hematologic Malignancies.

"Deciding which regimen to use has increasingly become a challenge," said Dr. Kumar. "The treatment paradigm for myeloma has evolved considerably, but it is starting to converge, irrespective of whether the patient is transplant-eligible or -ineligible."

\section{Newly Diagnosed MM}

In patients with newly diagnosed MM, the standard initial therapy for several years has been the combination of bortezomib, lenalidomide, and dexamethasone based on a SWOG trial that compared the triplet regimen with lenalidomide and dexamethasone alone (Figure 1). ${ }^{1}$ The addition of bortezomib to lenalidomide and dexamethasone not only improved the overall response rate (ORR), said Dr. Kumar, but also increased the depth of response in terms of complete responses. This translated to improved progression-free survival (PFS) and overall survival (OS) with the triplet versus the doublet, which made it a definitive therapy for newly diagnosed disease.

Many attempts have been made to improve on the combination of bortezomib/lenalidomide/dexamethasone, including switching bortezomib to carfilzomib, a nextgeneration proteasome inhibitor. However, results of the phase III ENDURANCE trial found no difference in PFS or overall response when comparing these 2 triplet regimens. ${ }^{2}$ Although the depth of response was higher with the carfilzomib regimen, said Dr. Kumar, this did not translate to better survival outcomes because of the toxicity profile of the combination.

Other attempts have been made to develop oral induction therapies. In a phase II trial, bortezomib was switched to ixazomib, an oral proteasome inhibitor, and the "all-oral" triplet regimen of ixazomib/lenalidomide/ dexamethasone was also effective, with an ORR $>80 \%$ and deepening responses with continued therapy. ${ }^{3} \mathrm{PFS}$ for the study cohort, which included transplant-eligible and -ineligible patients, was approximately 36 months, which is similar to the ENDURANCE trial, said Dr. Kumar.

Another approach to improve initial therapy for MM has been to switch the proteasome inhibitor to a different class of drug. The MAIA trial compared the addition of daratumumab, an anti-CD38 monoclonal antibody, to lenalidomide/dexamethasone versus lenalidomide/ dexamethasone alone in patients who were ineligible for transplant. ${ }^{4}$ Patients continued on this maintenance regimen until disease progression. The addition of daratumumab led to a significantly better ORR. Most importantly, said Dr. Kumar, it also significantly improved the complete response rate and rates of minimal residual disease (MRD) negativity. This led to an improved PFS, with a median PFS that has still not been reached versus a median PFS of 32 months for lenalidomide/dexamethasone.

"Clearly, the combination of a proteasome inhibitor with an immunomodulatory drug or a monoclonal 

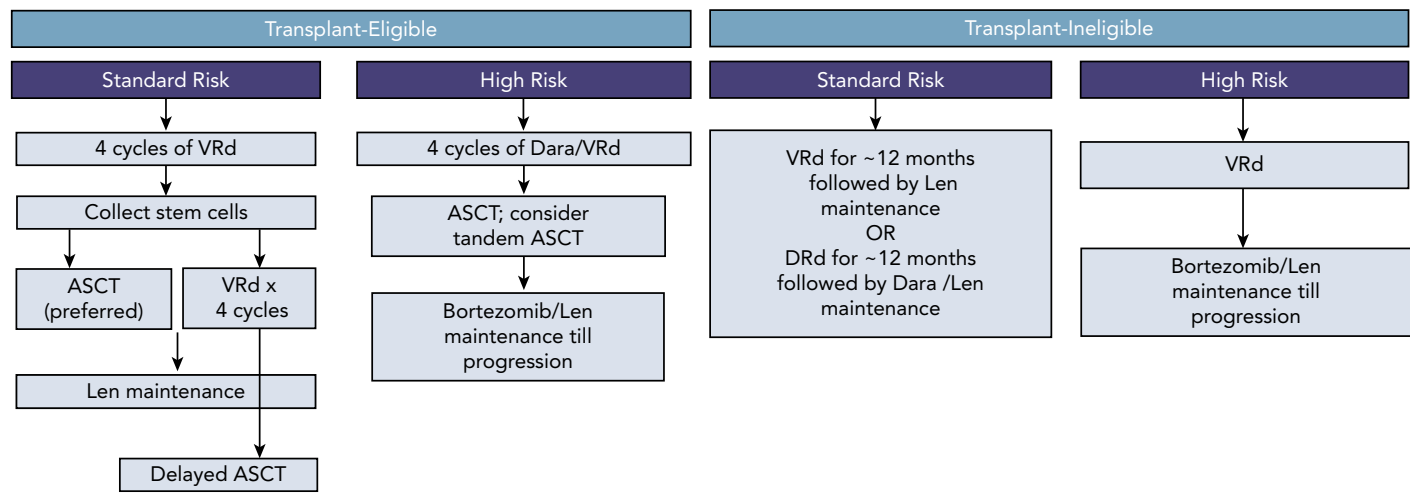

Figure 1. Approach to newly diagnosed multiple myeloma.

Abbreviations: ASCT, autologous stem cell transplantation; Dara, daratumumab; DRd, daratumumab/lenalidomide/dexamethasone; Len, lenalidomide; VRd, bortezomib/lenalidomide/dexamethasone.

antibody with an immunomodulatory drug has been an excellent strategy for achieving good disease response," said Dr. Kumar, who noted that the field has tried to improve on this triplet by adding an additional class of drug.

The CASSIOPEIA trial evaluated the addition of daratumumab to the triplet regimen of bortezomib/ thalidomide/dexamethasone before and after autologous stem-cell transplantation in patients with newly diagnosed MM. ${ }^{5}$ The addition of daratumumab improved depth of response and PFS with acceptable safety compared with bortezomib/thalidomide/dexamethasone alone.

The GRIFFIN trial used a similar study design with 2 main exceptions: lenalidomide was substituted for thalidomide and all patients were randomized to maintenance of either lenalidomide + daratumumab or lenalidomide alone. ${ }^{6}$ The addition of daratumumab to bortezomib/ lenalidomide/dexamethasone led to a $10 \%$ improvement in stringent complete response and a significant increase in the depth of response as indicated by MRD status.

Clinicians are awaiting data about the impact on OS of adding a fourth drug to the regimen from both the GRIFFIN and CASSIOPEIA trials, Dr. Kumar reported.

According to Dr. Kumar, continuing patients indefinitely on 4-drug regimens will be "extremely difficult from the standpoint of both toxicity and cost." The phase II MASTER trial investigated discontinuation of therapy based on the experience of MRD negativity. ${ }^{7}$ Patients received induction therapy with daratumumab/carfilzomib/ lenalidomide/dexamethasone, followed by autologous transplantation, followed by consolidation for 4 cycles, followed by additional consolidation for patients who did not reach MRD negativity. Those who tested negative for MRD on 2 occasions then underwent a treatment-free period with MRD surveillance.

"With an MRD-based consolidation treatment approach, nearly $95 \%$ of patients were able to achieve a stringent complete response," said Dr. Kumar. "More importantly, almost $63 \%$ of patients achieved MRD negative status. These results are quite impressive for the quadruplet but need to be replicated in large phase III trial settings," he added.

Another approach is to develop a quadruplet regimen with an oral proteasome inhibitor for greater convenience. A phase II trial of ixazomib + lenalidomide/dexamethasone/ daratumumab in patients with newly diagnosed myeloma demonstrated an excellent overall response. ${ }^{8}$ More than $90 \%$ of patients experienced a partial response or better, including a significant proportion of patients with a very good partial response. A second cohort that evaluated discontinuing dexamethasone early on did not show a significant difference in depth of response.

In patients with newly diagnosed MM who are ineligible for stem cell transplantation, the phase III ALCYONE trial showed that the addition of daratumumab to bortezomib/melphalan/prednisone prolonged OS. ${ }^{9}$ With $>3$ years of follow-up, the daratumumab arm continued to show significant improvement in PFS versus the triplet regimen, with no new safety concerns. According to Dr. Kumar, however, one disadvantage of this study was the complete discontinuation of therapy in the control arm compared with continued therapy in the quadruplet arm.

\section{Relapsed/Refractory MM}

As Dr. Kumar reported, significant changes have occurred in recent years in the setting of relapsed MM, including several phase III trials that have evaluated bortezomibbased and lenalidomide-based combinations (Figure 2). All of these regimens have shown significant improvement in PFS, said Dr. Kumar. Some studies have also shown improved OS, such as the ENDEAVOR trial, which studied carfilzomib/dexamethasone versus bortezomib/dexamethasone. ${ }^{10}$

Similarly, proteasome inhibitors such as carfilzomib and ixazomib and monoclonal antibodies such as 

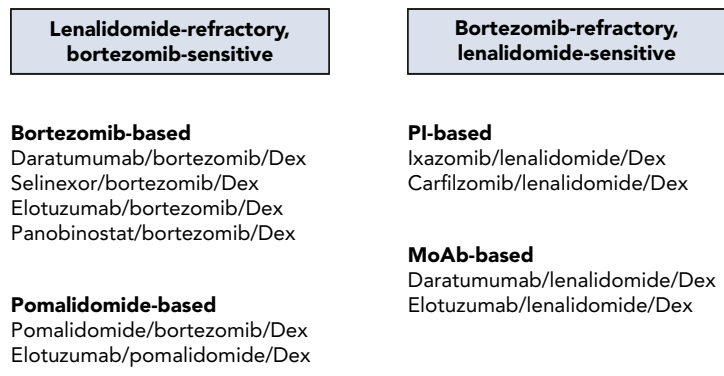

PI-based

Ixazomib/lenalidomide/Dex Carfilzomib/lenalidomide/Dex

MoAb-based

Daratumumab/lenalidomide/Dex Elotuzumab/lenalidomide/Dex

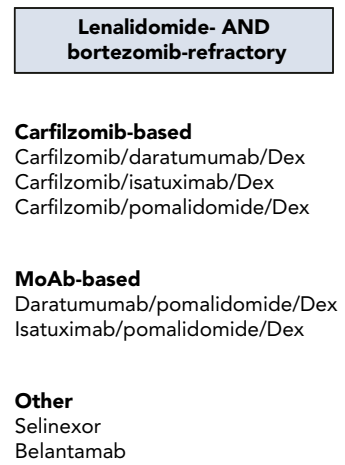

Figure 2. Selecting the optimal regimen.

Abbreviations: Dex, dexamethasone; MoAB, monoclonal antibody; PI, proteasome inhibitor.

daratumumab and elotuzumab have been combined with lenalidomide and dexamethasone and have shown significant efficacy as triplets, improving not only PFS but also OS in some trials.

According to Dr. Kumar, however, most patients seen in the United States at first relapse have been on lenalidomide maintenance or lenalidomide + bortezomib maintenance, making them refractory to one or both of these drugs. "Newer data would be very helpful, especially when considering changing the class of drug in these patients," he said.

The ICARIA trial, which randomized patients with relapsed and refractory myeloma to isatuximab + pomalidomide and low-dose dexamethasone versus pomalidomide and low-dose dexamethasone alone, showed significantly improved depth of response and a near doubling of PFS with the addition of isatuximab, an anti-CD38 monoclonal antibody. ${ }^{11}$ Although there was a trend toward improved OS, the data still need to mature, said Dr. Kumar.

Elotuzumab has also been combined with pomalidomide and dexamethasone in a randomized phase II trial that showed improved PFS versus pomalidomide and dexamethasone alone. ${ }^{12}$

In another phase II trial, the combination of daratumumab with pomalidomide and dexamethasone showed that $>70 \%$ of patients who are daratumumab-naïve and refractory to multiple lines of therapy can benefit from the addition of the monoclonal antibody to pomalidomide and dexamethasone. ${ }^{13} \mathrm{~A}$ phase III trial has been completed, but results are not available at this time, said Dr. Kumar.

Carfilzomib + pomalidomide and dexamethasone has also been studied in patients with multiple previous lines of therapy. Results of a phase II study showed median PFS of 7.2 months and median OS of 20.6 months. ${ }^{14}$

Another approach to patients whose disease is refractory to lenalidomide is to use a monoclonal antibody in combination with a proteasome inhibitor. The CANDOR trial showed that the triplet regimen of carfilzomib/ daratumumab/dexamethasone significantly prolonged
PFS versus carfilzomib/dexamethasone alone in patients with relapsed or refractory MM and was associated with a favorable risk-benefit profile. ${ }^{15}$

A similar trial was conducted with isatuximab, another monoclonal antibody. The IKEMA trial randomized patients to isatuximab + carfilzomib and dexamethasone versus carfilzomib and dexamethasone alone and showed improved ORR, depth of response, and MRD negativity, which translated to better median PFS. ${ }^{16}$

"Despite these advances, however, patients eventually become refractory to proteasome inhibitors, immunomodulatory drugs, and often to the monoclonal antibodies," said Dr. Kumar. "New classes of drug must then be considered."

A new drug that was recently approved for relapsed $\mathrm{MM}$ is selinexor, a nuclear transport protein inhibitor. In combination with dexamethasone, selinexor had a response rate of $26 \%$ in a population of patients with disease that was refractory to multiple lines of therapy. ${ }^{17}$ Moreover, OS and PFS appeared to be quite promising compared with historical data for triple-class refractory patients.

Selinexor has also been combined with bortezomib in a phase III trial that randomized patients to selinexor + bortezomib and dexamethasone versus bortezomib and dexamethasone alone. ${ }^{18}$ Results showed that the addition of selinexor to bortezomib and dexamethasone significantly improved PFS, despite bortezomib being given only weekly in the triplet compared with twice weekly in the doublet, said Dr. Kumar.

Finally, belantamab mafodotin, an antibody drug conjugate targeting B-cell maturation antigen, was recently approved for MM as a single agent. Results of the DREAMM-2 study showed an ORR of $34 \%$ in patients who were refractory to multiple lines of therapy, which translated to improved PFS and OS..$^{19}$ A recent combination trial showed an ORR of $78 \%$ in patients who had received more than one previous line of therapy when belantamab mafodotin was combined with bortezomib and dexamethasone..$^{20}$ 
"Clearly, this is a regimen that we need to see more data from in the near future," Dr. Kumar concluded.

Disclosures: Dr. Kumar has disclosed that he has received honoraria from BeiGene; grant/research support from AbbVie, Inc., Amgen Inc., Bristol-Myers Squibb Company, CARsgen Therapeutics, Celgene Corporation, Janssen
Pharmaceutica Products, LP, Kite Pharma, Medlmmune Inc., Merck \& Co., Inc., Novartis Pharmaceuticals Corporation, Roche Laboratories, Inc., sanofi-aventis U.S., Takeda Pharmaceuticals North America, Inc., TeneBio; and consulting fees from Cellectar Biosciences, GeneCentrix, Inc, Oncopeptides. He is also a scientific advisor for AbbVie, Inc., Amgen Inc., Celgene Corporation, Genentech, Inc., Janssen Pharmaceutica Products, LP, Medlmmune Inc., and sanofi-aventis U.S.

Correspondence: Shaji K. Kumar, MD, Mayo Clinic Cancer Center, 200 First Street SW, Rochester, MN 55905. Email: Kumar.shaji@mayo.edu

\section{References}

1. Durie BGM, Hoering A, Abidi $M H$, et al Bortezomib with lenalidomide and dexamethasone versus lenalidomide and dexamethasone alone in patients with newly diagnosed myeloma without intent for immediate autologous stem-cell transplant (SWOG S0777): a randomized, openlabel, phase 3 trial. Lancet 2017;389:519-527.

2. Kumar $S$, Jacobus SJ, Cohen $A D$, et al Carfilzomib, lenalidomide, and dexamethasone (KRd) versus bortezomib, lenalidomide, and dexamethasone (VRd) for initial therapy of newly diagnosed multiple myeloma: results of ENDURANCE (E1A11) phase III trial [published online June 01, 2020]. J Clin Oncol, doi: 10.1200/JCO.2020.38_suppl.LBA3

3. Kumar SK, Berdeja JG, Niesvizky R, et al Ixazomib, lenalidomide, and dexamethasone in patients with newly diagnosed multiple myeloma: long-term follow-up including ixazomib maintenance. Leukemia 2019; 33 1736-1746.

4. Facon $\mathrm{T}$, Kumar $\mathrm{S}$, Plesner $\mathrm{T}$, et al Daratumumab plus lenalidomide and dexamethasone for untreated myeloma. N Engl J Med 2019;380: 2104-2115.

5. Moreau P, Attal M, Hulin C, et al Bortezomib, thalidomide, and dexamethasone with or without daratumumab before and after autologous stem-cell transplantation for newly diagnosed multiple myeloma (CASSIOPEIA): a randomized, open-label, phase 3 study. Lancet 2019; 394:29-38.

6. Voorhees PM, Kaufman JL, Laubach J, et al Daratumumab, lenalidomide bortezomib, and dexamethasone for transplant-eligible newly diagnosed multiple myeloma: the GRIFFIN trial. Blood 2020;136:936-945.

7. Costa L, Chhabra S, Godby K, et al Daratumumab, carfilzomib, lenalidomide and dexamethasone (Dara-KRd) induction, autologous transplantation and post-transplant, response-adapted, measurable residual disease (MRD)-based Dara-Krd consolidation in patients with newly diagnosed multiple myeloma (NDMM) [abstract 653]. Presented at the 2019 American Society of Hematology Annual Meeting; December 7, 2019; Orlando, Florida.

8. Kapoor P, Gertz MA, Laplant B, et al Phase 2 trial of daratumumab, ixazomib, lenalidomide and modified dose dexamethasone in patients with newly diagnosed multiple myeloma [abstract]. Blood 2019;134(Suppl 1):Abstract 864

9. Mateos MV, Cavo M, Blade J, et al Overall survival with daratumumab, bortezomib, melphalan, and prednisone in newly diagnosed multiple myeloma (ALCYONE): a randomized, open-label, phase 3 trial. Lancet 2020;395:132-141.

10. Dimopoulos MA, Goldschmidt H, Niesvizky R, et al Carfilzomib or bortezomib in relapsed or refractory multiple myeloma (ENDEAVOR): an interim overall survival analysis of an open-label, randomised, phase 3 trial. Lancet Oncol 2017;18:1327-1337.

11. Attal M, Richardson PG, Rajkumar SV, et al Isatuximab plus pomalidomide and low-dose dexamethasone versus pomalidomide and low-dose dexamethasone in patients with relapsed and refractory multiple myeloma (ICARIA-MM): a randomised, multicentre, open-label, phase 3 study. Lancet 2019;394:2096-2107.

12. Dimopoulos MA, Dytfeld D, Grosicki S, et al Elotuzumab plus pomalidomide and dexamethasone for multiple myeloma. N Engl J Med 2018; 379:1811-1822.

13. Chari A, Suvannasankha A, Fay JW, et al Daratumumab plus pomalidomide and dexamethasone in relapsed and/or refractory multiple myeloma. Blood 2017;130:974-981.

14. Shah JJ, Stadtmauer EA, Abonour R, et al Carfilzomib, pomalidomide, and dexamethasone for relapsed or refractory myeloma. Blood 2015;126: 2284-2290.

15. Dimopoulos M, Quach H, Mateos MV, et al Carfilzomib, dexamethasone, and daratumumab versus carfilzomib and dexamethasone for patients with relapsed or refractory multiple myeloma (CANDOR): results from a randomised, multicentre, open-label, phase 3 study. Lancet 2020;396: 186-197.

16. Moreau P, Dimopoulos MA, Yong $K$, et al Isatuximab plus carfilzomib/ dexamethasone versus carfilzomib/dexamethasone in patients with relapsed/refractory multiple myeloma: IKEMA phase III study design. Future Oncol 2020;16:4347-4358.

17. Chari A, Vogl DT, Dimopoulos MA, et al Results of the pivotal STORM study (part 2) in penta-refractory multiple myeloma (MM): deep and durable responses with oral selinexor plus low dose dexamethasone in patients with penta-refractory MM [abstract]. Blood 2018;132(Suppl 1): Abstract 598.

18. Dimopoulos MA, Delimpasi S, Simonova M, et al Weekly selinexor, bortezomib and dexamethasone versus twice weekly bortezomib and dexamethasone in patients with multiple myeloma after 1-3 prior therapies: initial results of the phase III BOSTON study. Presented at the 2020 ASCO Virtual Scientific Program; May 29, 2020. Abstract 8501.

19. Lonial S, Lee HC, Badros A, et al Belantamab mafodotin for relapsed or refractory multiple myeloma (DREAMM-2): a two-arm, randomised, openlabel, phase 2 study. Lancet Oncol 2020;21:207-221.

20. Nooka AK, Stockerl-Goldstein $\mathrm{K}$, Quch $\mathrm{H}$, et al DREAMM-6: safety and tolerability of belantamab mafodotin in combination with bortezomib/ dexamethasone in relapsed/refractory multiple myeloma (RRMM) [abstract]. J Clin Oncol 2020;38(Suppl):Abstract 8502. 